\title{
Endometriosis apendicular. Reporte de caso
}

\author{
Gonzalo Perrone*, Martín Salvatierra†, Daniel Gonzálezł
}

\section{Resumen}

La endometriosis se define como la presencia de glándulas endometriales y/o estroma fuera de la cavidad uterina. Afecta a mujeres en edad fértil y su etiología es desconocida, aunque se reconoce un componente genético.

La endometriosis apendicular es una entidad infrecuente que se presenta como dolor abdominal crónico recurrente. Presentamos el caso de una paciente que consultó por dolor abdominal en fosa ilíaca derecha. Se realizó laparoscopía exploradora y apendicectomía cuyo análisis anatomopatológico informó endometriosis apendicular.

Palabras clave: Endometriosis apendicular Informe de casos

Key words: $\quad$ Appendiceal endometriosis Case reports

\section{Introducción}

La endometriosis se define como la presencia de tejido endometrial, consistente en glándulas y/o estroma, ubicado fuera del útero ${ }^{(1)}$.

La prevalencia de endometriosis en mujeres asintomáticas es desconocida. Existen revisiones sistemáticas que informan una prevalencia de $60 \%$ en pacientes de sexo femenino con dolor abdominal crónico recurren$\mathrm{te}^{(2)}$.

El compromiso del tracto gastrointestinal por endometriosis abdominopélvica ha sido reportado entre el 3\% a 37\% de las mujeres, siendo el colon sigmoides, recto e íleon terminal las vísceras más frecuentemente afecta$\mathrm{das}^{(3)}$.

La endometriosis apendicular es una entidad infrecuente formando parte de un hallazgo inusual menor al $1 \%$ en algunas series de apendicectomía ${ }^{(4)}$.

Algunos trabajos reportan una incidencia de $0,3 \%$ en piezas de apendicectomía ${ }^{(5)}$.

Collins, en $1955^{(6)}$, comunicó una prevalencia de $0,054 \%$ basándose en el análisis aleatorizado de más de 50.000 piezas de apendicectomía. Trabajos más recientes reportan una prevalencia de $0,05 \%$ en 2.047 piezas de apendicectomía ${ }^{(7)}$.

\section{Caso clínico}

Paciente de 37 años que consultó por dolor abdominal en fosa ilíaca derecha de 10 días de evolución. Se exacerba en las últimas 24 horas.

Náuseas. No vómitos. No fiebre.

Como antecedente personal refiere dolor abdominal recurrente en hemiabdomen inferior que ha requerido múltiples consultas.

Última menstruación: 15 días antes de la consulta actual.

Al examen físico dolor a la palpación y defensa en fosa ilíaca derecha.

\footnotetext{
* Residente de cirugía general. Clínica Quirúrgica 3. Hospital Maciel. Región Norte. Hospital Regional Salto, Salto, Uruguay. † Cirujano general. Clínica Quirúrgica 3. Hospital Maciel. Región Norte. Hospital Regional Salto. Salto, Uruguay.

‡ Prof. Clínica Quirúrgica 3. Hospital Maciel. Universidad de la República. Facultad de Medicina. Montevideo, Uruguay.

Departamento de Cirugía, Hospital Regional Salto, Salto-Uruguay. Clínica Quirúrgica 3, Hospital Maciel, Montevideo, Uruguay.

Correspondencia: Dr. Gonzalo Perrone. Correo electrónico: gonpedr@hotmail.com

Recibido: 29/8/18

Aprobado: $15 / 11 / 18$
} 


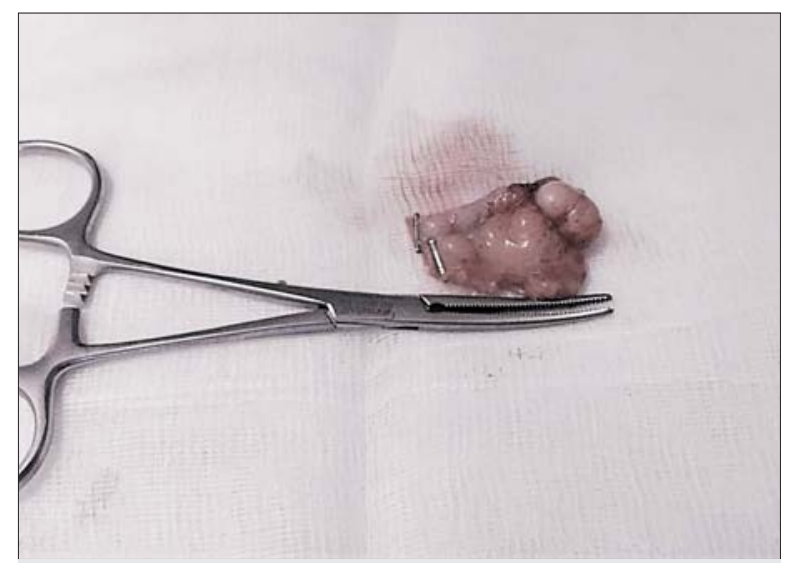

Figura 1. Pieza de apendicectomía.

Hemograma: 11.700 glóbulos blancos, 69\% neutrófilos. Hormona gonadotrofina coriónica humana (BHCG) negativa. Ecografía ginecológica sin alteraciones.

Con planteo preoperatorio de apendicitis aguda se realizó laparoscopía exploradora de urgencia. Exploración, apéndice edematoso. Se realizó la apendicectomía (figura 1).

Buena evolución posoperatoria. Alta al segundo día.

A los 11 meses de evolución, asintomática.

Anatomía patológica: sección de pared apendicular que muestra proceso fibrótico. Glándulas de aspecto endometrial con estroma y escasa hemorragia con los caracteres de endometriosis apendicular (figura 2).

\section{Discusión}

El dolor en fosa ilíaca derecha es un motivo de consulta frecuente, planteando diversos diagnósticos diferenciales en mujeres en edad fértil.

La endometriosis apendicular como causa de dolor abdominal es infrecuente. No encontramos para este trabajo un reporte nacional de prevalencia, siendo esta difícil de cuantificar.

La endometriosis apendicular puede ser asintomática y en los casos sintomáticos el diagnóstico preoperatorio es dificultoso ${ }^{(8)}$.

En el caso presentado se manifestó como dolor en fosa ilíaca y defensa localizada. La duda diagnóstica surgió a partir de los antecedentes de la enfermedad actual dados por episodios de dolor recurrente en fosa iliaca, así como el tiempo de inicio del dolor en el episodio actual.

Existe controversia con respecto a que estudio imagenológico presenta mayor rendimiento para el diagnóstico. En la literatura se mencionan diversos estudios de imagen como la ecografía transvaginal, resonancia magnética, ecografía transvaginal, con contraste salino, contraste de agua rectal y con preparación intestinal.

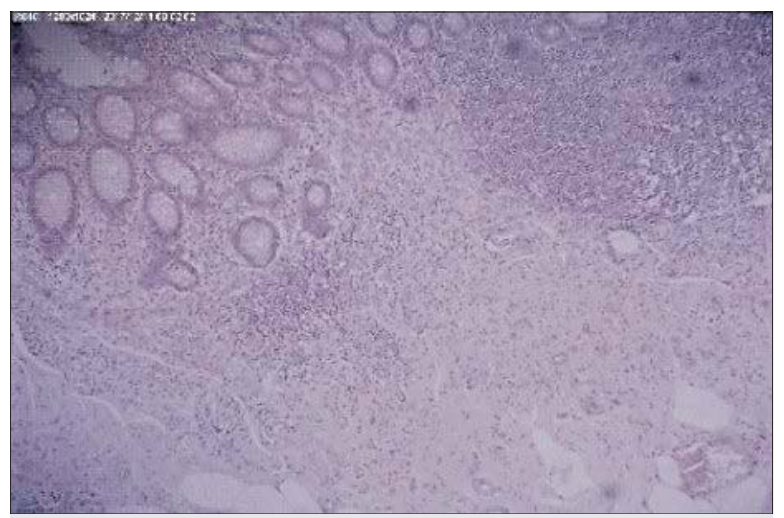

Figura 2. Aspecto microscópico de la pieza de apendicectomía.

Hasta la fecha, no hay indicaciones claras ni pruebas sólidas disponibles con respecto a la mejor técnica. Además, la elección de un método en lugar de otro sigue siendo "empírico" o basado en la experiencia perso$\mathrm{nal}^{(9)}$.

Actualmente, la laparoscopía sigue siendo el estándar de oro para el diagnóstico macroscópico. Sin embargo, el riesgo quirúrgico es mayor que los métodos imagenológicos, por lo cual la controversia es conocer cuál es el método ideal ${ }^{(10)}$.

Dada la incertidumbre diagnóstica de los métodos de imagen, la laparoscopía exploratoria con apendicetomía resulta mandatoria. Esto se debe a que el diagnóstico definitivo de endometriosis u otro proceso maligno apendicular debe ser confirmado con el estudio anatomopatológico de la pieza quirúrgica, siendo de capital importancia descartar una neoplasia mucinosa apendicular como diagnóstico diferencial ${ }^{(11,12)}$.

\section{Abstract}

Endometriosis is defined as the presence of endometrial glands and/or stroma outside the uterine cavity. It affects women of childbearing age, and its etiology is unknown, although a genetic component is recognized. Appendiceal endometriosis is an unusual condition which presents as chronic or recurrent abdominal pain. We present the case of a patient who consulted for abdominal pain in the right iliac fossa. Exploratory laparoscopy and appendectomy were performed, the histological analysis of which reported appendiceal endometriosis.

\section{Resumo}

Define-se endometriose como a presença de glândulas endometriais e/ou estroma fora da cavidade uterina. Afeta a mulheres em idade fértil, e sua etiologia não é conhecida, embora se reconheça um componente gené- 
tico. A endometriose apendicular é uma entidade pouco frequente que se apresenta com dor abdominal crônica recorrente. Apresentamos o caso de uma paciente que consultou por dor abdominal na fossa ilíaca direita. Realizou-se uma laparoscopia exploradora e apendicectomia cujo laudo anatomopatológico informou endometriose apendicular.

\section{Bibliografía}

1. Cobo Vázquez C, Marcacuzco Quinto A, Manrique Municio A, Justo Alonso I, Caso Maestro Ó, Calvo Pulido J, et al. Endometriosis apendicular. Nuestra experiencia en los últimos 11 años. Clin Invest Ginecol Obstet 2017; 45(4):151-6. doi: https://doi.org/10.1016/j.gine.2017.03.003.

2. Janssen E, Rijkers A, Hoppenbrouwers K, Meuleman C, D'Hooghe T. Prevalence of endometriosis diagnosed by laparoscopy in adolescents with dysmenorrhea or chronic pelvic pain: a systematic review. Hum Reprod Update 2013; 19(5):570-82.

3. Yantiss R, Clement P, Young R. Endometriosis of the Intestinal Tract: a study of 44 cases of a disease that may cause diverse challenges in clinical and pathologic evaluation. Am J Surg Pathol 2001; 25(4):445-54.

4. Akbulut S, Tas M, Sogutcu N, Arikanoglu Z, Basbug M, Ulku A, et al. Unusual histopathological findings in appendectomy specimens: a retrospective analysis and literature review. World J Gastroenterol 2011; 17(15):1961-70.
5. Chandrasegaram M, Rothwell L, An E, Miller R. Pathologies of the appendix: a 10-year review of 4670 appendicectomy specimens. ANZ J Surg 2012; 82(11):844-7.

6. Collins D. Endometriosis of the vermiform appendix; review of literature, with addition of nine new instances, one of which cause severe melena. AMA Arch Surg 1951; 63(5): 617-22.

7. Unver N, Coban G, Arýcý D, Buyukpýnarbasýli N, Gucin Z, Malya F, et al. Unusual histopathological findings in appendectomy specimens: a retrospective analysis of 2047 cases. Int J Surg Pathol 2018 [Epub ahead of print]. doi: $10.1177 / 1066896918784650$.

8. Sánchez Cifuentes A, Candel Arenas M, Albarracín Marín-Blázquez A. Intestinal endometriosis. Our experience. Rev Esp Enferm Dig 2016; 108(8):524-5.

9. Nezhat C, Li A, Falik R, Copeland D, Razavi G, Shakib A, et al. Bowel endometriosis: diagnosis and management. Am J Obstet Gynecol 2018; 218(6):549-62.

10. Carbognin G, Girardi V, Pinali L, Raffaelli R, Bergamini V, Pozzi Mucelli R. Assessment of pelvic endometriosis: correlation of US and MRI with laparoscopic findings. Radiol Med 2006; 111(5):687-701.

11. Tong Y, Chen Y, Zhu S. Ileocecal endometriosis and a diagnosis dilemma: a case report and literature review. World J Gastroenterol 2013; 19(23):3707-10.

12. Mitchell A, Dubé P, Sideris L. Dysplastic intestinal-type metaplasia of appendiceal endometriosis: a mimic of low grade appendiceal mucinous neoplasm. Diagn Pathol 2014; 9(1):39. 